\title{
INDICATEURS PALEOENVIRONNEMENTAUX ET ECONOMIE \\ RURALE \\ LE CAS DE LA GAULE NARBONNAISE \\ Par \\ PHILIPPE LEVEAU
}

Le statut de "province développée" est acquis par la Gaule Narbonnaise à partir de sa création comme province sénatoriale sous Auguste: un statut juridique consacre une évolution culturelle et un développement économique. À la fin du Ier s., Pline le dit explicitement: agrorum cultu, virorum morumque dignatione, amplitudine opum nulli provinciarum postferanda breviterque Italia verius quam provincia (Naturalis Historia 3.31). Les auteurs modernes en trouvent la confirmation dans un développement urbain qui a laissé des traces remarquables dans le paysage moderne, dans les équipements (réseau viaire et infrastructures portuaires) et dans le peuplement des campagnes. Toutes ces données font l'objet d'un consensus auprès des chercheurs. Cet état des connaissances qui avantage les villes explique l'importance prise dans les années 1980 par les études sur la ville antique, "ville de consommation". Rome, au premier chef, et, à son image, toutes les villes de l'Empire étaient le lieu de consommation de richesses sur l'origine et surtout sur le processus d'acquisition desquelles s'interrogent les historiens de l'économie antique.

Je ne reviens pas ici sur un débat qui a donné lieu à de vives discussions ${ }^{1}$. Ses termes sont commandés par la nature des indicateurs dont on dispose. Pour faire évoluer le paradigme ou en proposer un nouveau, il faut les modifier, c'est-à-dire mieux connaître ce qui se passe entre les sites urbains. Je tenterai donc de montrer comment la recherche archéologique met en évidence l'impact de Rome sur les économies rurales, soit, l'effet d'une économie commerciale sur la production agricole, sur les produits eux-mêmes et sur leur localisation.

\footnotetext{
'Ph. Leveau, 'La ville antique, "ville de consummation" ? (Parasitisme social et économie antique)', Études rurales 89-90-91 (1983), 275-289; H. M. Parkins, Roman urbanism. Beyond the consumer city (London/New York 1997).
} 


\section{Le développement économique de la Narbonnaise : l'enrichissement des connaissances, l'évolution des méthodes d'étude et le changement des paradigmes}

En principe, un tel projet nécessite un bilan de la recherche archéologique depuis une vingtaine d'années afin d'en souligner les avancées et l'apport à la question du développement économique. Il n'est pas possible de le mener à bien ici. Je me contente donc de rappeler pour mémoire le développement des recherches sur un certain nombre de sites majeurs, dont le mieux étudié est incontestablement Nîmes ${ }^{2}$. Mais on dispose également de remarquables synthèses sur les villes d'Aix-en-Provence ${ }^{3}$ et de Fréjus ${ }^{4}$. À l'inverse, d'autres villes souffrent d'un défaut de publications comme en particulier Marseille ou d'un défaut de recherches comme Narbonne. Les études sur le commerce sont évidemment essentielles. Leur développement a été favorisé par l'étude des céramiques, surtout par celle des conteneurs de produits agricoles, amphores et dolia, trouvés sur les sites de consommation ou provenant d'épaves. Il faut y ajouter les recherches en cours sur les ports antiques.

Dans le domaine rural, la nouvelle série des Cartes Archéologiques de la Gaule dirigée par M. Provost rend accessible pour certains secteurs géographiques une documentation archéologique d'une grande richesse. Le récent colloque sur les Campagnes de la fin de l'Antiquité apporte de riches bilans régionaux ${ }^{5}$. S'agissant de l'espace rural, la nouveauté est cette "archéologie du champ" que les archéologues développent depuis les années 1990, grâce aux moyens mis à leur disposition par les aménageurs et en réponse à un souhait anciennement formulé. Initiée en France du Nord, cette approche a été appliquée au T.G.V. Sud-Est, sur les tracés autoroutiers ${ }^{6}$

\footnotetext{
${ }^{2}$ On en trouvera une bibliographie dans le plus récent des volumes consacrés à ce site : P. Garmy \& M. Monteil, Le quartier des Bénédictins à Nîmes (Gard). Découvertes anciennes et fouilles 1966-1992 (Paris 2000).

${ }^{3}$ J. Guyon, N. Nin, L. Rivet, S. Saulnier, Aix-en-Provence (Montpellier 1998).

${ }^{4}$ L. Rivet, D. Brentchaloff, S. Roucole, S. Saulnier, Fréjus (Montpellier 2001).

${ }^{5}$ P. Ouzoulias, C. Pellecuer, C. Raynaud, P. van Ossel, P. Garmy, eds., Les campagnes de la Gaule à la fin de l'Antiquité. Actes du colloque de Montpellier (Antibes 2001).

${ }^{6} \mathrm{Ph}$. Boissinot \& J.-E. Brochier, 'Pour une archéologie du champ', dans G. Chouquer, Les formes $d u$ paysage 3 : L'analyse des systèmes spatiaux (Paris 1997), 35-56.
} 
et en archéologie urbaine ${ }^{7}$. Aux fouilles d'exploitations agricoles et de résidences rurales et aux fouilles de champs a été associé un remarquable développement de prospections archéologiques dont l'apport renouvelle les connaissances de l'occupation des campagnes en Gaule Narbonnaise.

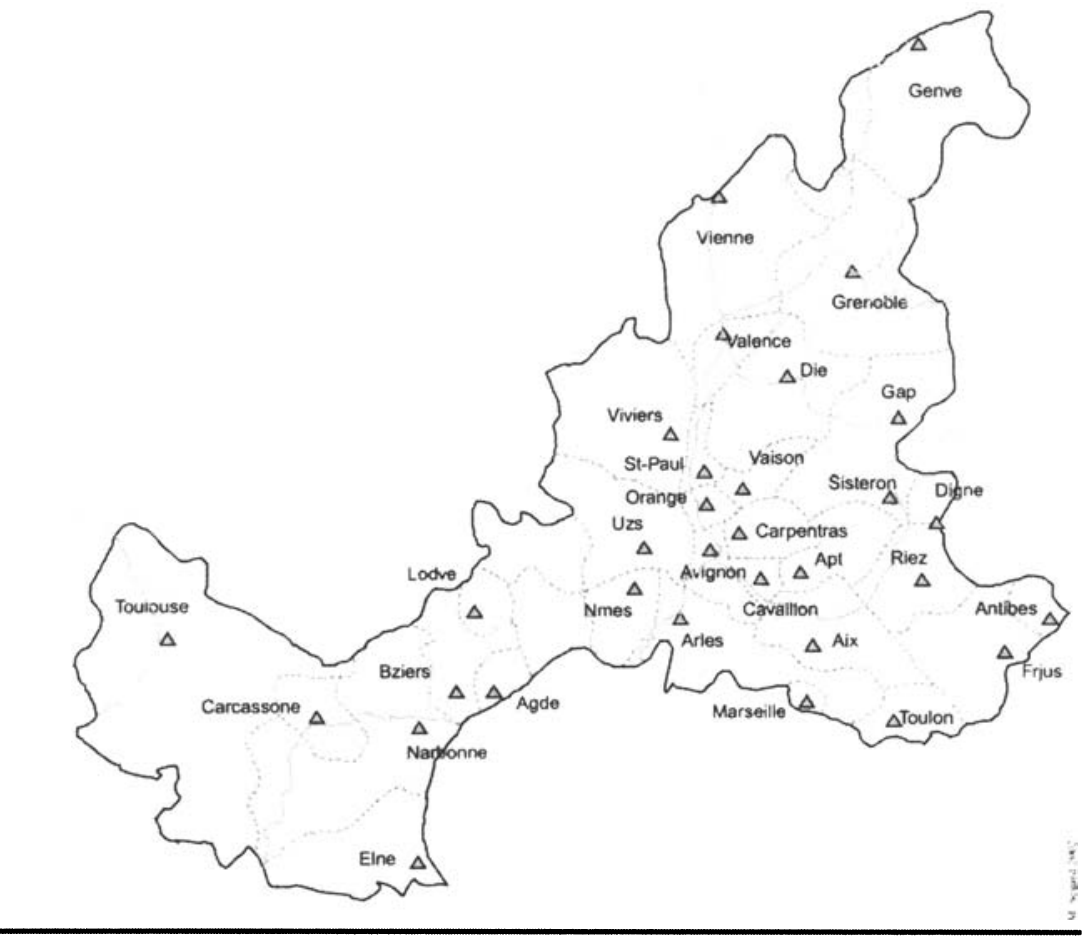

Province de Narbonnaise

Il faut accorder une mention particulière aux travaux qui ont été coordonnés par le programme européen Archaeomedes dans le Bas et le Moyen Rhône. Ce projet a fédéré un nombre important de chercheurs en particulier un groupe d'archéologues et de géographes qui a publié une première synthèse des résultats obtenus ${ }^{8}$. À partir de prospections portant sur plusieurs régions

${ }^{7}$ M. Monteil, S. Barberan, M. Piskorz, L. Vidal, 'Culture de la vigne et traces de plantation des Ile-Ier s. av. J.-C. dans la proche campagne de Nîmes (Gard)', Revue Archéologique de Narbonnaise 32 (1999), 67-123.

${ }^{8}$ F. Durand-Dastès, F. Favory, J.-L. Fiches, H. Mathian, D. Pumain, C. Raynaud, L. Sanders, S. van der Leeuw, Des oppida aux métropoles (Paris 1998). 
présentées comme des tests, ils ont élaboré des courbes quantifiées qui mettent en évidence les phases du développement de l'habitat antique sur le territoire de la cité romaine de Nîmes, en Languedoc oriental et dans la Vallée du Rhône. Pour cela, aux matériaux archéologiques de prospections, ils ont adapté des méthodes statistiques utilisées par les géographes en modélisation. Ces quantifications ouvrent la voie à des comparaisons interrégionales. Elles ont fait certes l'objet de critiques qui dénoncent l'irréalisme d'extrapolations fondées sur les seules observations de surface. Cependant, pour la Narbonnaise, on manque encore d'une réflexion de la qualité des remarques qu'ont présentées P. Ouzoulias et P. van Ossel pour l'Île-de-France ${ }^{9}$. Le progrès conceptuel n'en est pas moins incontestable par rapport aux approches anciennes qui accordaient une priorité à la répartition topographique et à la classification typologique. Perché à l'époque protohistorique, l'habitat serait descendu dans la plaine, puis il serait remonté sur les hauteurs à la fin de l'Antiquité. Son évolution aurait été caractérisée par un phénomène de dispersion se traduisant par la multiplication des habitats du type de la villa. Dans sa généralité, ce schéma d'évolution est juste. Mais il ne rend pas compte des différences de rythmes dans les dynamiques régionales du peuplement.

L'ensemble de ces méthodes a ouvert la voie à un renouvellement des études du développement rural en Gaule Narbonnaise. Jusqu'à ces dernières années, une place essentielle était accordée à la romanisation du paysage par la mise en place de vastes centuriations. Le schéma proposé était simple. À la rationalité planimétrique de la ville coloniale correspondait une organisation tout aussi rationnelle et régulière de l'espace rural assurée par la centuriation. Ainsi, pour reprendre une expression de G. Duby, Rome aurait jeté sur la Gaule du Sud, un vaste filet, la centuriation, division rationnelle et géométrique de l'espace. Enregistrée par l'autorité romaine sur des cadastres, - dont celui d'Orange apporte un témoignage unique et capital一, elle était réputée avoir offert un cadre à l'appropriation du sol provincial par de riches propriétaires fonciers issus de l'émigration civile et militaire romaine et italienne ou par des indigènes ralliés à Rome. Fondateurs des villes, ils en contrôlaient les campagnes et en orientaient la production vers une économie de profit. Dans ce cadre, une relation simple était établie entre la centurie, division romaine de l'espace, et la villa, centre d'exploitation

\footnotetext{
${ }^{9}$ P. Ouzoulias \& P. van Ossel, 'Dynamiques du peuplement et formes de l'habitat tardif: le cas de
} l'île-de-France' 147-172, dans Ouzoulias et alii 2001, op. cit. (n.5), 225-246. 
domaniale et lieu de résidence de l'aristocratie urbaine. La majorité des archéologues adhéraient à ce qui était devenu un paradigme.

Dans les années 1990, ce "paradigme cadastral" a été remis en question; l'on assiste maintenant à sa "déconstruction". G. Chouquer, l'un des principaux chercheurs français travaillant sur ce thème, vient de publier un ouvrage qui marque un renoncement à la tentative de décrire l'appropriation du sol par Rome à partir de l'étude des parcellaires fossiles ${ }^{10}$. Il refuse désormais d'associer une forme du paysage à une période précise et dénonce une confusion entre organisation des surfaces et polarisation des réseaux de communication par un lieu central: cette dernière, qui n'est pas spécifiquement médiévale, explique la forme étoilée prise par les terroirs. Cette position s'appuie sur des considérations théoriques, en particulier une reconnaissance de l'autonomie des systèmes spatiaux (les formes du paysage) par rapport aux systèmes sociaux (Rome, la société médiévale, ...) et aux causalités historiques (la conquête militaire entraînant une réorganisation massive des surfaces pour laquelle le processus le plus simple est la division géométrique). Il reconnaît que Rome n'avait ni opéré sur un espace vide ni sous-estimé la résistance des modes antérieurs d'occupation du sol.

L'ensemble de ces données est maintenant complété par l'apport d'une archéologie écologique qui bénéficie de l'essor des sciences de l'environnement : les disciplines liées à l'écologie proprement dite et celles qui ont pour objet l'étude du sédiment. En Gaule du Sud, leur utilisation par les archéologues travaillant sur les périodes historiques ne remonte pas à plus d'une quinzaine d'années. Longtemps, les géomorphologues n'ont collaboré qu'avec les pré et proto historiens. La prise en compte de leurs études pour la période antique est liée à l'élargissement de la notion de site archéologique et au développement de la prospection. Considéré d'abord comme un masque du site archéologique, le sédiment a maintenant acquis un statut d'objet archéologique, utilisé pour l'identification de la mise en culture des sols.

Les études portant sur la pollution des écosystèmes par les métaux lourds permettent la caractérisation d'activités artisanales et industrielles. Actuellement le meilleur exemple est fourni par la pollution par le plomb identifiée dans les glaces du Groenland. Enregistrant la variation des rejets de plomb dans l'atmosphère, elles apportent un témoignage sur les activités

${ }^{10} \mathrm{Cf} \mathrm{Ph}$. Leveau, Critique de G. Chouquer, L'étude des Paysages. Essai sur leurs formes et leur histoire (Paris1999), dans Histoire et Sociétés rurales 15 (2001), 238-243. 
minières et métallurgiques liées à ce métal et à l'extraction de l'argent qui lui est associé. Pendant une période s'étendant entre 500 avant J.-C. et 200 environ, la concentration en plomb augmente de 5 fois par rapport à la concentration naturelle. Un retour à des niveaux presque naturels se produit vers 500. L'origine anthropique de ce plomb est démontrée par le rapport isotopique ${ }^{206} \mathrm{~Pb} /{ }^{207} / \mathrm{Pb}$ et une relation avec l'essor de la métallurgie antique apparaît évidente. Ces enregistrements témoignent d'un phénomène mondial et ne nous renseignent pas sur la part du monde méditerranéen. Mais, bien entendu, l'analyse de prélèvements effectués dans des dépôts permet de passer du global au local, de mettre en évidence la production minière ou des activités de plomberies sur un site précis ${ }^{11}$.

\section{Les indicateurs du développement économique dans les paléo- environnements : le cas de la Vallée du Rhône}

L'apport de ces approches à l'évaluation de l'impact de l'économie romaine en Gaule du Sud peut être montré à partir de travaux qui ont été réalisés en Vallée du Rhône où les conditions d'observations sont particulièrement favorables ${ }^{12}$. La vallée compte en effet un nombre important de villes romaines importantes qui la jalonnent entre Arles et Lyon. La majorité ont accédé au statut de colonies de droit romain. Certaines ont une origine militaire : Arles, Orange, Valence et Lyon. Mais pas toutes: Vienne et Avignon doivent à leur importance leur promotion à ce titre envié. Dans le cas de Vienne, il s'agit d'une capitale de cité dont les recherches archéologiques restituent l'importance. Celui d'Avignon est différent : la ville est ancienne, mais ce n'était pas une capitale. Dans tous les cas, le rôle du fleuve et de sa vallée, axe majeur du commerce de l'Occident romain, est fondamental. L'étude de l'organisation de l'espace rural bénéficie de l'apport d'un document exceptionnel, les marbres d'Orange.

Longtemps l'exploitation de cette documentation a été limitée par un milieu difficilement appréhendé par les archéologues, la vallée fluviale. Les spécificités de son évolution rendent un tel milieu peu propice à la recherche archéologique. Dans la plaine, l'alluvionnement a été parfois considérable, masquant les vestiges de l'habitat et des activités agricoles. Sur le fleuve, les

${ }^{11}$ F.B. Pyatt, 'An imperial legacy ? An exploration of the environmental impact of ancient metal mining and smelting in southern Jordan', Journal of Archaeological Science 27 (2000), 771-778; K.A. Hudson-Edwards \& M.G. Macklin, 'Medieval lead pollution in the River Ouse at York, England', Journal of Archaeological Science 26 (1999), 809-819.

${ }^{12} \mathrm{Ph}$. Leveau, 'Le Rhône romain. Dynamiques fluviales, dynamiques sociales', Gallia 56 (1999), 1175. 
variations latérales du chenal entraînent l'érosion et la destruction des aménagements de berge, donc des traces d'aménagement portuaire. Les quelques découvertes archéologiques effectuées dans le lit fluvial datent plutôt du siècle dernier. En revanche, nous savons très peu de choses de celles auxquelles ont nécessairement donné lieu les travaux considérables qui, entre 1950 et 1980 , ont été réalisés pour la maîtrise du fleuve ou de l'aménagement autoroutier de sa vallée. À cela s'ajoutent des problèmes liés à l'organisation de l'archéologie française: le Rhône est une limite administrative, ce qui gêne le développement d'une archéologie du fleuve. Cependant cette situation ne présente pas que des désavantages. L'alluvionnement auquel sont soumis les fonds de vallée assure la conservation des travaux d'aménagement d'un milieu qui présente d'intéressantes potentialités agricoles. L'inondation y apporte des alluvions qui, mêlées aux sols par les labours, en assurent la fertilité. Ces terres sont donc riches à condition d'en contrôler l'hydrologie, ce qui peut être réalisé par l'implantation de réseaux de drainage. En principe en effet, sous les niveaux d'abandon qui les scellent, les fossés que l'on retrouve sont plutôt des fossés de drainage que d'irrigation.

Le progrès récent des connaissances relatives à l'exploitation agricole de la vallée ${ }^{13}$ est lié à deux opérations de nature différente menées l'une, dans la plaine d'Arles ${ }^{14}$, dans le cadre de l'archéologie programmée, l'autre, dans la moyenne Vallée du Rhône dans celui de l'archéologie préventive la construction de la ligne nouvelle du TGV. Dans ce cas, les archéologues ont pu disposer des moyens matériels d'étude permettant la réalisation de sondages profonds. Dans le cas de la Vallée du Rhône, les archéologues attendaient du paléo-environnement deux types de réponses. La question de l'extension et des caractéristiques des terres agricoles (avaient-elles besoin d'aménagements et lesquels ?) était plutôt posée au géomorphologue. L'importance de l'érosion est fonction de caractéristiques climatiques et de l'activité agricole. Cette dernière est susceptible de libérer les particules des sols que transportent ensuite le vent et les eaux. La seconde question portait sur les productions agricoles et la place de ces activités dans le paysage ; elle s'adressait au paléobotaniste auquel il était demandé d'évaluer l'importance

${ }^{13}$ B. Ode \& T. Odiot, 'L'habitat rural de la moyenne vallé du Rhône aux IVe et Ve siècles', dans Ouzoulias et alii 2001, op.cit. (n.5), 225-246.

${ }^{14} \mathrm{Ph}$. Leveau, 'Dynamiques environnementales et dynamiques sociales sur le territoire d'Arles antique', dans F. Vermeulen \& M. de Dapper, eds., Géoarchéologie des paysages de l'antiquité classique, Colloque International, Gand, 23-24 octobre 1998. Bulletin van de Vereniging Antieke Beschaving (BABESCH), supplement 5 (Leiden 2000), 105-118. 
et des caractéristiques du couvert forestier à partir de la proportion des pollens d'arbres dans les diagrammes polliniques et des données de l'anthracologie.

\section{II.1. L'apport des travaux géoarchéologiques dans le Tricastin et dans la plaine d'Orange}

L'apport du paléo-environnement a été remarquablement bien montré par le géoarchéologue J.-F. Berger, dans une série d'articles où il présente l'apport des travaux qu'il a conduits dans la plaine alluviale du Rhône Moyen, le Tricastin au nord, la plaine d'Orange au sud. Des fouilles pratiquées sur 132 sections de fossés permettent de suivre l'histoire des réseaux drainants, des curages auxquels a donné lieu leur entretien et, pour la fin de l'Antiquité, celle de leur ennoiement sous les dépôts de crues répétées. Ce travail a pour objectif final une modélisation paléoclimatique et, de ce fait, ne présente pas pour eux-mêmes les cas étudiés. Mais J.-F. Berger a accordé un intérêt particulier aux enregistrements de l'impact de l'agriculture d'époque romaine $^{15}$. Il montre d'abord l'inégale lisibilité des surfaces. Elle est évidemment en relation avec des recouvrements d'épaisseur inégale selon le secteur. Ils sont faibles parfois. Mais, dans la plaine d'Orange, leur importance est de l'ordre de $2,5 \mathrm{~m}$. Une étude pluridisciplinaire des sites de références montre la complexité de l'interprétation. Celle-ci doit tenir compte de ce que les phases les mieux conservées sont évidemment les phases d'abandon durant lesquelles les fossés ne sont pas curés.

Nous retiendrons l'apport de ces travaux pour deux faits essentiels pour notre propos. Le premier est l'identification d'un réseau d'irrigation par l'analyse de son comblement sédimentaire. Celui-ci prouve la continuité de l'écoulement et permet d'identifier l'origine du captage des eaux. Dans le cas d'un drainage, le sédiment du comblement proviendrait des roches locales. Aux Bartras ${ }^{16}$, la présence de gravillons de molasse tertiaire indique que le captage se trouvait à l'amont dans un contexte lithologique différent. On est donc en présence d'un réseau attribuable à la grande irrigation. Ceci constitue une nouveauté complète pour la Gaule du Sud. L'irrigation y est attestée depuis les périodes les plus anciennes de l'agriculture. Mais il s'agit de ce que l'on qualifie de petite irrigation : l'eau est captée dans une source proche ou dans un petit cours d'eau pour assurer des cultures saisonnières

\footnotetext{
${ }^{15} \mathrm{~J}$.-F. Berger, 'Les fossés bordiers historiques et l'histoire agraire rhodanienne', Etudes rurales 153154 (2000), 59-90.

${ }^{16}$ Berger 2000, op. cit. (n.15), 71.
} 
dans des jardins le plus souvent proches d'un lieu d'habitat. Cette forme d'irrigation doit être distinguée de la grande irrigation qui nécessite la construction d'installations permettant de recueillir, de conserver et de répartir l'eau pour les cultures à l'échelle d'une vallée ou d'une plaine, soit le franchissement d'un seuil. En Méditerranée pour l'époque antique ${ }^{17}$, celleci n'est attestée de manière certaine que dans les régions les plus sèches de l'Empire, là où les conditions écologiques l'imposaient: les marges désertiques, en Égypte en particulier, mais ailleurs également, ainsi dans le sud de la péninsule Ibérique où l'on connaît une série de barrages. Elle a aussi été pratiquée probablement en Italie ou en France du Sud, là où les conditions économiques le justifiaient. Les savoirs techniques étaient possédés en effet comme en attestent les grands aqueducs urbains romains. Des ouvrages célèbres comme ceux de Nîmes et d'Arles ont été construits pour satisfaire les besoins en eau d'une agglomération et non pour irriguer les campagnes. Dans le cas des aqueducs d'Arles, on observe qu'un ouvrage d'abord conçu pour une ville a subi une modification afin d'utiliser une partie des eaux pour les moulins ; cependant un usage principal de l'eau pour la force motrice n'en excluait évidemment pas l'utilisation secondaire pour l'irrigation du fond de la vallée à Barbegal ${ }^{18}$. Dans le cas de régions où une agriculture sèche était praticable, leur mise en œuvre pour l'agriculture doit être prouvée. Ainsi, en Provence et en Languedoc oriental, on considérait que la grande irrigation s'est développée seulement à l'époque moderne: en basse Provence, au XVI ${ }^{\mathrm{e}}$ s., l'objectif premier des constructeurs du canal de Craponne était encore la force motrice et non l'irrigation qui fut réalisée seulement par suite.

Le second fait mis en évidence porte sur l'évolution du paysage agricole. J.-F. Berger en a présenté les principes dans une étude conduite en Vallée du Rhône dans le Tricastin et la plaine d'Orange pour un secteur concerné par le cadastre romain. À cette occasion, il a clairement montré l'apport de l'analyse des remplissages sédimentaires de fossés pour l'archéologie agraire. Un arrêt de mise en culture entraîne une modification observable sur les coupes géomorphologiques. Les sédiments de crues ne sont plus mélangés aux labours. Un litage est alors visible. Les fossés qui ne remplissent plus leur fonction sont comblés. Une prairie protectrice s'installe

\footnotetext{
${ }^{17} \mathrm{P}$.-L. Viollet, L'hydraulique dans les civilisations anciennes, 5000 ans d'histoire (Paris 2000).

${ }^{18} \mathrm{Ph}$. Leveau, 'Les moulins romains de Barbegal, les ponts-aqueducs du vallon des Arcs et l'histoire naturelle de la vallée des Baux (Bilan de six ans de fouilles programmées)', Comptes Rendus de l'Académie des Inscriptions et Belles-Lettres (1995), 115-144.
} 
et bloque ou ralentit les apports de sédiments. Le remplissage des fossés est en outre caractérisé par une malachofaune hydro et hygrophile particulièrement abondante ${ }^{19}$.

À proximité de Lapalud où l'on situe le locus gromae du cadastre B d'Orange, aux Girardes, les archéologues ont eu l'occasion de fouiller un site rural - une villa? - et les champs situés à proximité et d'étudier une zone humide proche. Les études naturalistes qui ont porté sur celle-ci ont montré qu'à la charnière des $\mathrm{I}^{\mathrm{er}}$ et $\mathrm{II}^{\mathrm{e}} \mathrm{s}$., le drainage avait connu une période d'arrêt suivie d'un développement des friches. Opérée sur près de $20 \mathrm{ha}$, la fouille des champs avait montré que, durant la période précédente, les parcelles étaient cultivées en vigne ${ }^{20}$. Cette spécialisation agricole s'explique par le très fort développement de la viticulture mis en évidence dans l'ensemble de la Narbonnaise depuis quelques années. D'une manière générale, pour la même période, les prospections archéologiques démontrent un développement considérable du peuplement: les créations de sites sont nombreuses. Au tournant des $\mathrm{I}^{\mathrm{er}}$ et $\mathrm{II}^{\mathrm{e}}$ s., intervient un changement qui est caractérisé par l'arrêt des créations de site et par celui de la viticulture. L'archéologie du champ a permis une observation analogue un peu partout où cela était possible, c'est-à-dire en zone basse.

La diminution du nombre des sites occupés qui s'affirme à partir du milieu du $\mathrm{II}^{\mathrm{e}} \mathrm{s}$. a été interprétée en terme de "crise". Cette notion mérite d'être précisée en distinguant bien crise des milieux et crises des sociétés pour ces dernières, le pluriel est de rigueur. En effet, en réaction contre les généralisations précédentes, les archéologues adoptent une attitude plus prudente et tentent de confronter documentation archéologique et documentation épigraphique. Dans le cas du Rhône Moyen, bien documenté par le cadastre d'Orange, et par les travaux archéologiques dont il vient d'être question, la relation entre les deux crises n'est pas simple. Le cadastre est un document fiscal qui indique des limites que l'on croyait systématiquement matérialisées par des fossés. La réalité révélée par l'archéologie est complexe. Ph. Boissinot précise qu'il " $\mathrm{n}$ 'a pas systématiquement servi d'appui aux parcelles romaines qui traversent certains axes majeurs" "21. Mais l'archéologie du champ n'est évidemment que l'une des approches de l'exploitation de terroirs. Toute généralisation

\footnotetext{
${ }^{19}$ Berger 2000, op.cit. (n.15), 75.

${ }^{20} \mathrm{Ph}$. Boissinot, 'La trace des paysages agraires. L'archéologie des façons culturales en France', Etudes rurales 27-29 (2000), 23-38.

${ }^{21}$ Boissinot 2000, op.cit. (n.20), 26.
} 
doit être précédée d'une réflexion sur la conservation des faits archéologiques: les fosses de plantation sont conservées en secteur de plaine à la suite de recouvrement alluviaux alors que la vigne est considérée habituellement comme une culture de colline. À proximité de Lattes, les naturalistes qui en avaient mis en évidence la culture situaient les vignobles sur les pentes de collines proches; les fouilles de Port Ariane ont montré que la vigne était cultivée dans un secteur actuellement occupé par un marais. Les faits ne sont pas contradictoires. À Nîmes, les traces d'un vignoble avaient été préservées par des constructions d'époque augustéenne ${ }^{22}$. En fait l'archéologie rurale révèle la diversité de situations individuelles que l'on doit se garder de généraliser à l'ensemble d'un espace. Le développement de la prairie n'est pas nécessairement lié à une déprise agricole.

\section{2. La paléobotanique}

On manque encore de données polliniques susceptibles de montrer l'impact de la romanisation en moyenne Vallée du Rhône. Mais l'intérêt de cette approche a été démontré à l'aval dans le Bas-Rhône et la zone deltaïque pour lesquels on dispose d'une documentation écrite et archéologique importante du fait de l'existence de la colonie romaine d'Arles. À la lumière des états les plus "anciens" (fin du Moyen Âge et Temps Modernes) connus par des textes ou par des documents cartographiques, les historiens avaient imaginé que les paysages du Bas-Rhône dans l'Antiquité étaient dominés par l'eau. En fonction de cet état du milieu, on imaginait un développement urbain fondé essentiellement sur le commerce par le Rhône. Pour compenser le manque de terres dans la proche campagne, le pouvoir romain aurait attribué à la colonie des terres situées très à l'est. Une telle situation ne cadrait pas avec le schéma habituel d'une colonisation dont l'objectif principal était d'établir des vétérans. Arles était donc atypique ${ }^{23}$.

Les études conduites sur la partie orientale de la plaine et dans la vallée des Baux en collaboration avec les géomorphologues et les palynologues ont montré que les anciennes restitutions du paysage étaient erronées. Durant la période antique, le milieu se prêtait à la conquête agricole. Au prix de travaux dont on peut trouver des preuves régionales, les terres humides des basses plaines arlésiennes ont pu être drainées et rendues utilisables pour

\footnotetext{
${ }^{22}$ Monteil et alii 1999, op. cit. (n.7).

${ }^{23} \mathrm{Ph}$. Leveau, 'Colonie romaine et milieu naturel : Arles et les plaines du Bas-Rhône', dans L'homme et la dégradation de l'environnement. XVes Rencontres Internationales d'Archéologie et d'Histoire d'Antibes, 20-21-22 oct. 1994 (Juan-les-Pins 1995), 245-262.
} 
l'agriculture. On constate donc une cohérence entre le paysage antique restitué par les géomorphologues et l'image que se fait l'historien d'une terre où les Romains installent une colonie. Dans cette opération, les études de paléobotaniques confirment des hypothèses faites sur la mise en culture de ces terres et des précisions pour l'identification des productions agricoles et leur "spatialisation". Les deux secteurs économiques concernés sont la céréaliculture et l'élevage. La place majeure occupée par les céréales dans la production agricole est difficilement documentée par l'archéologie. Les sources écrites sont peu nombreuses et d'interprétation ambiguë. Actuellement quelques analyses polliniques justifient déjà l'hypothèse de terres à blé dans la région d'Arles et en Camargue ${ }^{24}$ et autorisent à supposer que la production de céréales ait connu une forte extension et qu'elle a constitué une des bases de la prospérité de la colonie romaine d'Arles. Si aucune date précise n'a pu être obtenue dans la vallée des Baux, à quelques kilomètres de là, en bordure de la plaine du Rhône, le profil de La Calade montre, pour le second âge du Fer et l'époque romaine, une courbe continue de Cerealia. Cerealia sp. domine; mais le seigle est présent et les plantes adventices (Centaurea solstitialis et Polygonum aviculare) sont irrégulièrement attestées.

Au début des années 1990, des bergeries antiques (bâtiments de grande taille allongés pointe orientée vers le mistral) ont été découvertes en prospection dans la plaine de Crau ${ }^{25}$. Dans cette remarquable découverte, la véritable nouveauté consiste non dans la présence du bétail en Crau, connue par Strabon (Géographie 4.1.7) et par Pline (Naturalis Historia 21.57) - mais dans la forme d'un élevage nécessitant la construction de bergeries. Des éleveurs romains (on pense aux nouveaux colons italiens) auraient entrepris d'élever des bêtes plus fragiles qui séjournaient dans la Crau en une période où il était nécessaire de les abriter dans des bergeries. Les auteurs ont envisagé que ces troupeaux aient été envoyés l'été dans les Alpes, comme ce fut le cas au Moyen Âge. Mais compte tenu de l'incertitude des dénombrements et des objections techniques au déplacement des troupeaux sur de longues distances, il est plus vraisemblable que ceux qui séjournaient en Crau durant l'hiver trouvaient,

\footnotetext{
${ }^{24}$ V. Andrieu-Ponel, Ph. Ponel, H. Bruneton, Ph. Leveau, 'Palaeoenvironments and cultural landscape of the last 2000 years reconstructed from pollen and coleopteran record in the Lower Rhône Valley, southern France' The Holocene 10,3 (2000), 341-355.

${ }^{25}$ O. Badan, J.-P. Brun, G. Congès, 'Les bergeries romaines de la Crau d'Arles. Les origines de la transhumance en Provence', Gallia 52 (1995), 263-310.
} 
l'été, de quoi subsister dans les zones humides de la plaine du Rhône, en Camargue et dans celles de la plaine d'Arles et de ses annexes comme la vallée des Baux. Le profil pollinique de la Calade en a apporté une confirmation. Dans ce cas, les études paléo-écologiques (palynologie, entomologie) mettent en évidence l'impact local de l'élevage dans les zones humides $^{26}$. L'hypothèse d'une origine antique de la transhumance n'est non plus validée par les études en cours sur l'occupation pastorale dans les Alpes: dans les diagrammes polliniques, la rupture apparaît médiévale et non antique ${ }^{27}$.

\section{3. Les aménagements du chenal fluvial}

Le cas de la plaine d'Orange avait permis d'évoquer l'origine agricole de la richesse consommée dans la ville. La récente découverte de l'épitaphe d'un sevir augustalis vient à point nommé pour nous rappeler qu'Orange était aussi en relation avec l'artère commerciale du Rhône. Elle entre dans une série jusqu'ici documentée pour les seules villes de Lyon et Arles que Strabon qualifie d'emporia. Le sévirat occupé par ce personnage à Orange et à Lyon s'explique vraisemblablement par des activités commerciales dans les deux colonies que lie le Rhône. L'importance de son mausolée permet de lui reconnaître une place importante dans des milieux d'affaires que l'on rencontre pour la première fois à Orange. Son patron Titus Pompeius Reginus devait appartenir à une puissante famille notable dont on imagine mal qu'elle n'ait pas eu un rapport avec le commerce régional ${ }^{28}$.

Strabon (4.1.14) souligne l'importance prise par le fleuve et sa vallée, comme axes de circulation, en relation avec l'ouverture de la Gaule au commerce méditerranéen que favorise la conquête; la découverte de matériel amphorique dans la vallée de la Saône en est la traduction au plan archéologique. Ce rôle est vérifié par les sources écrites dont les plus anciennes concernent la construction d'un canal d'accès au fleuve par Marius en 102 av. J.-C. La mise en vente par Caligula du mobilier de ses palais, à Lyon, en 40 suppose que l'on avait pu l'acheminer rapidement ${ }^{29}$. Le développement de l'axe fluvial a des conséquences sur l'organisation de la

\footnotetext{
${ }^{26}$ Andrieu-Ponel et alii 2000, op.cit. (n.24).

${ }^{27}$ Thèse de palynologie de M. Court-Picon en cours.

${ }^{28}$ V. Faure, J. Gascou, J.-M. Mignon, J. Planchon, S. Zugmeyer, 'Un sévir augustal d'Orange et de Lyon', Revue Archéologique de Narbonnaise 32 (1999), 21-30.

${ }^{29}$ D. van Berchem, Les routes et l'histoire, Études sur les Helvètes et leurs voisins dans l'Empire romain (Genève 1982), 108.
} 
Vallée du Rhône. Dans le couloir rhodanien, Rome favorise l'émergence de cités aux territoires de taille réduite sans doute aux dépens des grandes confédérations tribales Arécomiques à l'ouest, Salyens au sud-est et Voconces à l'est dont, au $\mathrm{III}^{\mathrm{e}}$ s. av. J.-C., les territoires incluaient des sections de la vallée.

La conquête romaine a entraîné un essor de la navigation fluviale sur un fleuve dont la dangerosité est un fait établi. Dans les premières années de leur occupation de la Gaule du Sud, les Romains ont aménagé l'accès au Rhône par le creusement d'un canal à l'est du delta, les fosses mariennes. Cette opération qui répondait d'abord à un problème stratégique (faciliter l'entrée du fleuve aux bateaux ravitailleurs de l'armée de Marius), prit une dimension économique: les Romains en firent don à leurs alliés marseillais qui "en retirèrent un grand profit par les taxes perçues sur les transports remontant et descendant le fleuve" (Strabon, ibid.). Les marbres d'Orange fournissent un exemple des aménagements du chenal réalisés par les ingénieurs romains. Dans ses travaux sur le moins bien conservé d'entre eux, le cadastre C, F. Salviat avait mis en évidence l'existence d'une fossa augusta, un canal de navigation d'une largeur d'une trentaine de mètres (fragment 351) que l'on situe maintenant dans la plaine d'Orange, comme le pensait déjà A. Piganiol. Les recherches géoarchéologiques de J.-L. Ballais et de J.-Cl. Meffre ont permis d'en préciser la fonction : permettre à la navigation de contourner une zone où le cours du Rhône était encombré d'îles rendant difficile la circulation sur le fleuve. En ce secteur du fleuve, caractérisé par l'existence d'un seuil et l'apport sédimentaire d'affluents, des îles se font et se défont. Recouvrant les galets d'une nappe würmienne, le fleuve dépose des limons et crée un terroir riche pour qui en maitrise l'hydraulique ${ }^{30}$.

\section{Anthropisation du milieu et développement économique : "méditerranéisation" du climat et "front pionnier"}

Mises en série, les informations qu'apporte l'analyse archéologique des sites (approche "stationnelle") permettent d'accéder à des situations générales qui, dans le cas qui nous occupe, caractérisent le développement économique induit par l'intégration de la Gaule du Sud dans l'Empire. L'impact de ce développement dans les paléo-environnements correspond à ce que les environnementalistes qualifient d'anthropisation; ils y reconnaissent précisément des degrés séparés par des seuils : le seuil néolithique, le seuil

${ }^{30}$ Leveau 1999, op.cit. (n.12). 
gréco-romain, le seuil de la société industrielle. Durant les périodes antiques, l'environnement a incontestablement subi les effets d'activités de type industriel ; évoquée plus haut, la plomberie en donne un bon exemple. Mais, pour la période antique, le principal agent de l'anthropisation est encore l'activité agricole. L'impact de celle-ci sur le milieu n'est pas fondamentalement différent de celui d'un phénomène climatique. Ainsi les défrichements et le développement d'espaces pâturés sont susceptibles d'avoir sur les environnements des effets analogues à ceux d'une aridification du climat. L'interprétation de l'ambiguiité observée au niveau des impacts nécessite la collaboration de naturaliste et de l'archéologue.

Ce fait est au cœur d'une discussion qui divise les paléobotanistes, celle qui porte sur l'installation du climat méditerranéen, celui qui intéresse pratiquement l'ensemble de la Narbonnaise. Climat de transition, il est caractérisé par la sécheresse estivale, l'irrégularité et éventuellement la violence des précipitations automnales, ce qui fragilise le rapport entre ce milieu et les sociétés. Le palynologue français G. Jalut et des palynologues espagnols $^{31}$ ont proposé une reconstruction des processus qui ont accompagné cette mise en place. Ils prennent pour point de départ la constatation suivante : avant la période néolithique $(6000 \mathrm{BP})$, dans le nord de la Méditerranée, la végétation arbustive est caractérisée par la chênaie sempervirente et les forêts d'arbres à feuilles caduques. À partir de ce moment se développe une végétation sclérophylle et xérophile. Cette modification serait produite selon un gradient latitudinal sud-nord. Selon eux, entre $40^{\circ}$ et $44^{\circ}$ de latitude nord, soit dans la région qui nous intéresse, une modification dans la répartition annuelle des précipitations aurait conduit à l'installation de la sécheresse estivale caractéristique du climat méditerranéen entre 3300 et 1000 B.P., soit donc entre l'âge du Bronze et la période carolingienne. Plus précoce en Espagne du Sud, la mise en place de la sécheresse estivale aurait débuté vers 2600-1900 B.P. (2850-1630 cal. B.P.) dans le Golfe du Lion. Selon les auteurs d'une série d'analyses polliniques portant sur les sites du Nord-Est de la Péninsule Ibérique, malgré l'essor de l'urbanisation en Catalogne à l'époque romaine à la fin du second

\footnotetext{
${ }^{31}$ G. Jalut, A. Esteban Amat, S. Riera y Mora, M. Fontugne, R. Mook, L. Bonnet et T. Gauquelin, 'Holocene climatic changes in the western Mediterranean: installations of the Mediterranean climate', Comptes Rendus de l'Académie des Sciences. Sciences de la terre et des planètes 325 série IIa (Paris 1997), 327-334.
} 
âge du Fer, le climat reste le principal facteur des changements observés dans les environnements ${ }^{32}$. Il en irait de même en Languedoc occidental.

Mais l'augmentation des capacités d'intervention et la complexité croissante des sociétés rendent délicate l'interprétation des données environnementales en termes d'histoire du milieu. Un consensus existe à propos des faits eux-mêmes: à partir de l'âge des métaux, une tendance climatique, -la progression de la sécheresse-, accompagne le développement de foyers culturels remontant du sud de la Péninsule ibérique vers les côtes méditerranéennes françaises. Mais, ce que G. Jalut interprète comme l'effet d'une aridification liée à la mise en place du climat méditerranéen est attribué par d'autres à l'anthropisation ${ }^{33}$. Pour A. Pons et P. Quezel, la "méditerranéisation" du climat observable à partir de la fin de l'âge du Fer a donc une composante culturelle essentielle. Le développement du chêne vert au détriment du chêne à feuilles caduques n'est pas l'indicateur d'un changement climatique; il résulte de l'anthropisation du couvert végétal.

Les différences que les études paléoenvironnementales font apparaître en Languedoc traduisent les inégalités régionales du développement économique. Les palynologues ont fait deux observations: dans le bassin de l'Aude, la diminution des taux d'essences mésophiles est plus accentuée et plus précoce tandis que, d'une manière générale, le démarrage des déboisements est plus rapide que sur le littoral du Languedoc oriental où les marqueurs botaniques de milieux ouverts n'atteignent leurs maxima qu'après le Haut Moyen Âge. Ainsi, à l'ouest de l'Hérault, la fin de la Protohistoire et le début de la romanisation sont marqués par une chute des pollens arboréens caractérisant un très fort déboisement. Le phénomène n'a certainement pas une origine climatique. Il est probablement lié à la proximité de Narbonne, capitale de la Province, et à une urbanisation régionale beaucoup plus forte que sur le littoral du Languedoc oriental où Nîmes, le principal centre romain, est situé à l'intérieur. Dans les zones littorales catalane et française, la différence entre les territoires de Barcino et de Tarragona, entre la région de Lattes et celle de Narbonne, a, dans les deux cas, pour origine la présence

\footnotetext{
${ }^{32} \mathrm{~F}$. Burjachs, M. Blech, D. Marzoli, R. Julià, 'Evolución del paisaje vegetal en relación con el uso del territorio en la edad del Hierro en el NE de la Peninsula Ibérica', dans Els productes alimentaris d'origen vegetal a l'edat del ferro de l'Europa occidental: de la produccio al consum. XXIIe colloque international pour l'étude de l'Age du Fer, Monografies del Museu d'Arqueologia de CatalunyaGirona 18 (2000), 31-42.

${ }^{33}$ A. Pons \& P. Quezel, 'À propos de la mise en place du climat méditerranéen', Comptes Rendus de l'Académie des Sciences 327 série IIa (Paris 1998), 755-760.
} 
de deux capitales provinciales romaines. Plus qu'aucun facteur climatique, le développement économique régional explique la précocité relative et l'importance des agressions observées sur le milieu naturel aux abords de celles-ci.

Ce n'est donc pas un hasard si des archéologues travaillant dans la longue durée ont appliqué à la romanisation de la Gaule Narbonnaise un concept qui offre un remarquable parallèle avec celui de la "méditerranéisation", le concept de "front pionnier". Les données archéologiques recueillies en prospection ont fait l'objet de traitements statistiques autorisant à définir des gradients de développement dans l'occupation du sol. Ce concept a servi à caractériser l'évolution de l'occupation des campagnes en Vallée du Rhône à l'époque romaine ${ }^{34}$. Pour un géographe, le front pionnier est la limite atteinte par des colons défrichant des terres faiblement peuplées. Pour un historien, il évoque la conquête agricole des grandes plaines de l'Amérique du Nord. Les préhistoriens ont utilisé cette expression pour caractériser les processus de néolithisation; elle évoque alors un développement économique associé à un changement culturel. S'agissant de la Vallée du Rhône où l'occupation pré-romaine est ancienne et importante, l'emploi de cette expression peut être admis à condition de bien en préciser les conditions d'emploi. S'agissant de la Vallée du Rhône et de l'époque romaine, les échelles de temps d'un tel front pionnier différent de celles de la néolithisation de l'Ancien Monde et les échelles d'espaces de celles de la conquête des grandes plaines de l'Ouest du Nouveau Monde. La plus importante différence est l'existence de poches où subsistent les formes économiques anciennes. En Gaule Narbonnaise en général et en Vallée du Rhône en particulier, la colonisation romaine entraîne l'implantation de pôles urbains dont l'isolement relatif au milieu des tribus ou confédérations tribales gauloises justifie au Ier s. encore l'emploi d'une autre image soulignant l'hétérogénéité des situations, celle d'une "peau de léopard".

Pour la Gaule Narbonnaise, l'intérêt d'une échelle d'observation micro régionale et le changement de perspective qu'elle introduit dans l'évaluation de l'impact économique de la romanisation sont illustrés par le cas des moulins de Barbegal. Il y a une cinquantaine d'années, pour interpréter cette usine, F. Benoit se plaçait à l'échelle de l'économie de la province. Selon lui, les cryptoportiques du forum d'Arles auraient été des horrea recevant les blés amenés par la route et la voie fluviale. Ces blés auraient été amenés de

${ }^{34}$ Durand-Dastès et alii 1998 (n.8), 104-106. 
ces entrepôts à Barbegal pour être moulus et distribués à la troupe et à la population de la région. Il s'appuyait sur les travaux de Rostovtzeff ${ }^{35}$. Dans cette reconstruction qui reste largement admise, la question de l'approvisionnement était appréhendée à l'échelle de l'Empire. Depuis, on a montré que ces moulins étaient bien incapables de jouer le rôle qui leur était attribué: leur production n'excédait pas les besoins en farine de la ville d'Arles. Le point de vue adopté est celui qui a été présenté plus haut: démontrer par une étude micro régionale que les sols alluviaux de la plaine d'Arles produisaient des céréales qui étaient commercialisés hors du territoire de la ville ou utilisées pour l'approvisionnement de la ville.

\section{Conclusion}

Ce qui a été montré est inspiré par une idée centrale: l'amélioration des connaissances permet de modifier ou de préciser les paradigmes. Elle-même est étroitement liée aux méthodes et techniques d'approches. Dans le cas présent, la paléo-écologie me paraît porteuse de l'espoir d'une documentation nouvelle sur les économies antiques. Appliquée à une micro région, elle permet de voir si le vide de la documentation écrite et de la documentation archéologique correspond à une réalité de l'occupation humaine ou bien la masque. C'est un moyen d'identifier les forts contrastes existant entre des espaces plus développés et plus performants au plan économique et d'autres restés en marge.

Il faut abandonner un schéma évolutionniste simple conçu comme un progrès conduisant de la "protohistoire" à la "période romaine". Grâce au développement interne et aux influences hellénistiques, en Gaule du Sud, les bases de l'économie agricole sont acquises durant la protohistoire: plantes cultivées, animaux élevés, outils, techniques de culture, maîtrise des sols (drainage, irrigation, construction de terrasses). La nouveauté principale apportée par Rome réside dans l'intégration de la région à l'économie commerciale de l'Empire. Mais le progrès général dissimule de fortes disparités et, à l'échelle micro régionale, deux économies agricoles coexistent, une économie paysanne poursuivant la tradition protohistorique et une économie organisée en fonction du profit ("capitalistique"). Pas plus qu'une autre, la Gaule du Sud n'est une province homogène. Le recours au concept d'hétérogénéité spatiale permet d'intégrer l'opposition entre monde indigène et monde romain. L'intégration administrative d'une zone

\footnotetext{
${ }^{35}$ F. Benoit 'L'usine de meunerie hydraulique de Barbegal (Arles)', Revue Archéologique 15,1 (1940), 71 n.1.
} 
géographique dans la Provence ne se traduit pas nécessairement par une utilisation des modes de gestion de l'espace que l'on rencontre dans les secteurs les plus développés - les territoires des fondations coloniales romaines par exemple-. La Gaule Narbonnaise est bien comme le dit Pline Italia verius quam provincia, mais comme dans les autres provinces, s'y juxtaposent des formes économiques irréductibles.

Aix-en-Provence, novembre 2001 